\title{
Osteopontin delays resolution of liver fibrosis
}

\author{
Tung-Ming Leung ${ }^{1}$, Xiaodong Wang ${ }^{1}$, Naoto Kitamura ${ }^{1}$, Maria I Fiel ${ }^{2}$ and Natalia Nieto ${ }^{1}$
}

To date, considerable progress has been made both in the mechanisms driving liver fibrosis and in the prevention of disease progression. Resolution of liver fibrosis is an emerging field in hepatology; yet, the mediators involved remain elusive. Earlier work from our laboratory demonstrated that the matricellular cytokine osteopontin (OPN) is pro-fibrogenic by promoting hepatic stellate cell (HSC) activation and extracellular matrix (ECM) deposition in vitro and in vivo and specifically by governing fibrillar collagen-I expression, the key pro-fibrogenic protein. Here we hypothesized that OPN could also delay the resolution of liver fibrosis by sustaining collagen-I synthesis or by preventing its degradation. To demonstrate this, wild-type (WT) and OPN-knockout $\left(\mathrm{Opn}^{-/-}\right)$mice were administered thioacetamide (TAA) in the drinking water for 4 months. Half of the mice were killed at 4 months to assess the extent of fibrosis at the peak of injury, and the rest of the mice were killed 2 months after TAA withdrawal to determine the rate of fibrosis resolution. Following TAA cessation, livers from $\mathrm{Opn}^{-/-}$mice showed no centrilobular and parenchymal necrosis along with faster ECM remodeling than WT mice. The latter was quantified by less fibrillar collagen-I immunostaining. Western blot analysis demonstrated a significant decrease in fibrillar collagen-I and in tissue inhibitor of metalloproteinase-1 (TIMP-1) in $\mathrm{Opn}^{-1-}$ mice undergoing fibrosis resolution compared with WT mice. In conclusion, these results suggest that OPN delays liver fibrosis resolution due to sustained fibrillar collagen-I deposition; hence, inhibiting OPN could be an effective therapeutic strategy for resolving liver fibrosis.

Laboratory Investigation (2013) 93, 1082-1089; doi:10.1038/labinvest.2013.104; published online 2 September 2013

KEYWORDS: collagen-l; extracellular matrix; hepatic fibrosis; hepatic stellate cells; osteopontin

Cirrhosis, the end stage of hepatic fibrosis, occurs in almost all chronic liver diseases. Among the risk factors contributing to the pathophysiology of liver fibrosis are viral hepatitis, alcohol abuse, drugs and toxins, non-alcoholic fatty liver disease, obesity and insulin resistance, metabolic disorders, and autoimmune disease. All these stimuli and medical conditions cause significant liver damage over time and trigger a wound-healing response that involves activation of HSCs, significant ECM deposition (mostly fibrillar collagen-I), active ECM remodeling, and replication of hepatocytes to compensate for the loss of liver mass. ${ }^{1}$

The ECM is particularly dynamic and undergoes constant turnover during wound healing. Well-coordinated regulation of ECM remodeling is essential to maintain homeostasis and prevent disease. ${ }^{2}$ Fibrosis results from the imbalance between excessive ECM accumulation and insufficient remodeling after sustained liver injury with pathological fibrillar collagen buildup as end point. Recent literature in the field suggests that hepatic fibrosis is reversible particularly after cessation of the causative factor. ${ }^{3,4}$ Indeed, this has been demonstrated both in humans and in rodents; ${ }^{5-7}$ however, there is still insufficient knowledge on the mediators that could condition fibrosis resolution. Thus, there is a pressing need to identify them to develop new therapies to promote fibrosis resolution.

Activated HSCs are a key source of ECM in the injured liver. ${ }^{8}$ Under physiological conditions or even under mild liver injury, the degradation of fibrillar collagen is wellcoordinated by the activity of matrix metalloproteinases (MMPs) and tissue inhibitor of metalloproteinases (TIMPs) produced by these cells and whose expression increases both in fibrosis and in cirrhosis. ${ }^{9}$

Decreased activity of TIMPs', apoptosis of HSCs, and even reversal of activated HSCs to a more quiescent state are suggested as key events for fibrosis resolution. ${ }^{10,11}$ As activated HSCs are a major source of fibrillar collagens and of TIMPs, clearance of HSCs could facilitate matrix remodeling. Indeed, several studies have demonstrated that clearing HSCs

\footnotetext{
${ }^{1}$ Division of Liver Diseases, Department of Medicine, Mount Sinai School of Medicine, New York, NY, USA and ${ }^{2}$ Department of Pathology, Mount Sinai School of Medicine, New York, NY, USA

Correspondence: Dr N Nieto, Pharm D, PhD, Division of Liver Diseases, Department of Medicine, Mount Sinai School of Medicine, Box 1123, 1425 Madison Avenue, Room 11-70, New York, NY 10029, USA.

E-mail: natalia.nieto@mssm.edu

Received 21 June 2013; revised 30 July 2013; accepted 30 July 2013
} 
reverses fibrosis. ${ }^{7,12-14}$ Moreover, TIMP-1 has been shown to prevent HSCs apoptosis through the induction of B-cell lymphoma 2 (Bcl-2). ${ }^{13,15}$ Transgenic mice with liver-specific overexpression of TIMP-1 treated with carbon tetrachloride display delayed fibrosis resolution compared with WT mice. Similarly, recombinant TIMP-1 inhibited HSC apoptosis by lowering caspase- 3 activity. ${ }^{16}$

OPN is a matricellular cytokine highly involved in tissue remodeling. Previous work from our group showed that OPN is increased in drug-induced liver injury and fibrosis ${ }^{17}$ and that $\mathrm{Opn}^{-1-}$ mice were protected from hepatic fibrosis compared with WT mice. As we demonstrated that OPN has direct effects on fibrillar collagen-I, ${ }^{17}$ we hypothesized that OPN could also delay fibrosis resolution, perhaps independently of the aforementioned mechanisms. Thus, the aim of the present study was to compare the rate of fibrosis resolution after chronic TAA treatment in WT mice and in $\mathrm{Opn}^{-1-}$ mice with particular emphasis on fibrillar collagen-I expression, the key protein involved in liver fibrosis.

\section{MATERIALS AND METHODS General Methodology}

Details on general methodology such as H\&E staining and gelatine zymography to detect pro-, intermediate, and active MMP-2 and MMP-9 were described in previous publications from our laboratory. ${ }^{18-23}$

\section{Mice}

$\mathrm{Opn}^{-/-}$mice (B6.Cg-Spp1 $\left.1^{\text {tmlBlh}} / \mathrm{J}\right)$ and their matching WT littermates (C57BL/6 J) were obtained from the Jackson Laboratories (Bar Harbor, Maine). A targeting vector containing the neomycin-resistance cassette and the Herpes simplex virus thymidine kinase gene was used to disrupt exons 4 through 7 of the targeted gene. The targeted mutation deleted the coding region of the Opn gene. ${ }^{24}$ The resulting chimeric animals were backcrossed to C57BL/6 J for 10 generations. Mice were generated by intercrossing $\mathrm{Opn}^{+/-}$ mice, and littermates were used in all experiments.

\section{Induction of Liver Injury}

10-week-old male $\mathrm{Opn}^{-1-}$ mice and their WT littermates were used in this study. Liver injury was induced by treatment with $300 \mathrm{mg} / \mathrm{l}$ of TAA (Sigma, St Louis, MO) in the drinking water for 4 months. Controls received iso-volumetric amounts of water. Half of the mice were killed $48 \mathrm{~h}$ after TAA withdrawal to avoid acute liver injury. The rest of the mice were allowed recovery for 2 months before killing them. All animals received humane care according to the criteria outlined in the 'Guide for the Care and Use of Laboratory Animals' prepared by the National Academy of Sciences and published by the National Institutes of Health. All protocols were approved by the Institutional Animal Care and Use Committee at the Mount Sinai School of Medicine.

\section{Pathology}

In all experiments, the entire left liver lobe was collected, fixed in 10\% neutral-buffered formalin, and processed into paraffin sections for H\&E analysis, Sirius red/fast green or immunohistochemical (IHC) staining. Blind analysis according to the Brunt classification was used to determine the pathology scores and was done by a hepatopathologist at Mount Sinai School of Medicine. Inflammation was noted to be lymphocytes present in the lobules and were scored as follows: $1=$ rare foci, $2=$ up to five foci, and 3 when there were $>5$ foci. Centrilobular necrosis and parenchymal necrosis were each separately scored. The scores for centrilobular necrosis were $1=$ hepatocyte necrosis affecting only zone $3,2=$ in addition to zone 3 necrosis, occasional bridging necrosis was seen, and $3=$ pronounced bridging and confluent necrosis. Parenchymal necrosis was noted to be spotty necrosis or apoptosis in zones 2 and 1 . The scores for parenchymal necrosis were $1 \leq 1$ focus, $2=5-10$ foci, and 3 $\geq 10$ foci at $\times 100$ magnification. Ductular reaction was noted to be proliferation of bile ductules at the margins of the portal tracts and the score was $1=$ rare bile ductules present, 2 =irregular buds of bile ductules affecting some portal tracts, and $3=$ when bile ductules are more prominent and affect the majority of portal areas and/or strings of bile ductular epithelial cells that were seen intermingled with hepatocytes. The degree of fibrosis ranged from 0 to 4 and patterned after the Brunt system. ${ }^{25}$ Briefly, this was as follows: $1=$ perisinusoidal/perivenular fibrosis alone, $2=1$ plus portal fibrosis, $3=$ bridging fibrosis, and $4=$ cirrhosis. The assessment of the above scores was uniformly performed under $\times 100$ magnification.

\section{Immunohistochemistry}

The Collagen-I antibody was from Millipore (Billerica, MA), and the $\alpha$-SMA antibody was from Sigma. Reactions were developed using the Histostain Plus detection system from Invitrogen (Carlsbad, CA). For the collagen-I morphometric assessment, the integrated optical density (IOD) was calculated from 10 random fields per section containing similar size of portal tracts or hepatic veins at $\times 100$ magnification and using the Image-Pro 7.0 Software (Media Cybernetics, Bethesda, MD). The results were averaged and expressed as fold-change over controls. The rate of collagen-I clearance was expressed as the percentage of collagen-I-positive stained area in the recovered livers ( 2 months after cessation of TAA treatment) compared with that from mice killed at 4 months of TAA treatment.

\section{Western Blot}

Details on the Western blot methodology can be found in previous work from our laboratory. ${ }^{18-23}$ The antibodies to detect TIMP-1 and MMP-13 were purchased from Calbiochem (Darmstadt, Germany) and Millipore, respectively. The ECL reaction was developed using the Las 4000 
a
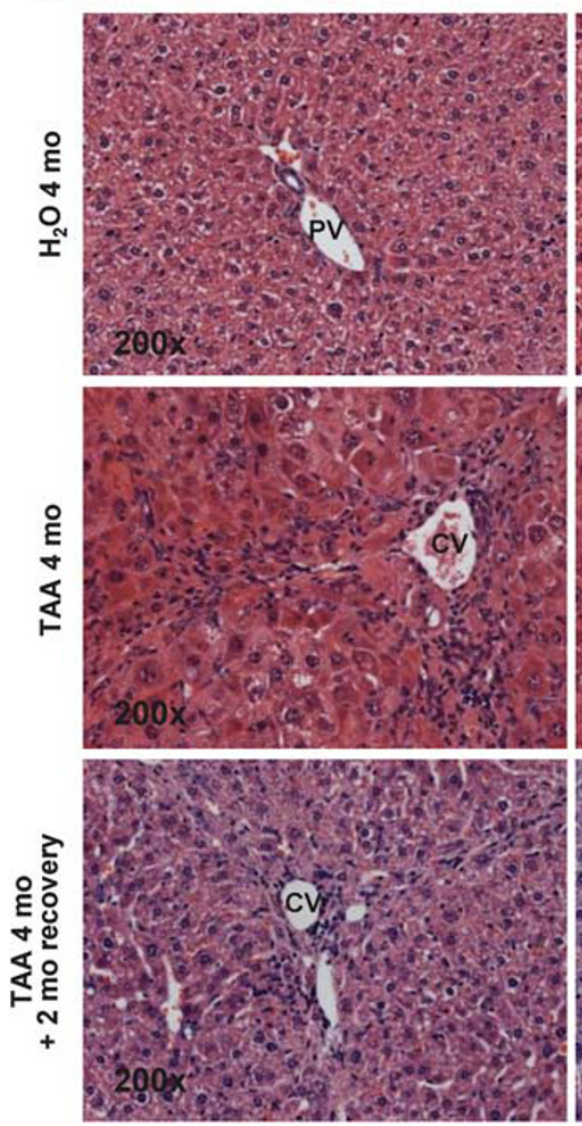

e

\begin{tabular}{cccc} 
& \multicolumn{3}{c}{ Percentage of clearance in 2 mo } \\
\cline { 2 - 4 } & Necrosis & Inflammation & Ductular Reaction \\
\hline WT & $50 \pm 10.2$ & $46 \pm 1.8$ & $73 \pm 7.8$ \\
Opn ${ }^{-/}$ & $69 \pm 7.8^{* \bullet}$ & $28 \pm 8.0$ & $49 \pm 4.2$ \\
\hline
\end{tabular}

$O p n^{\prime-}$
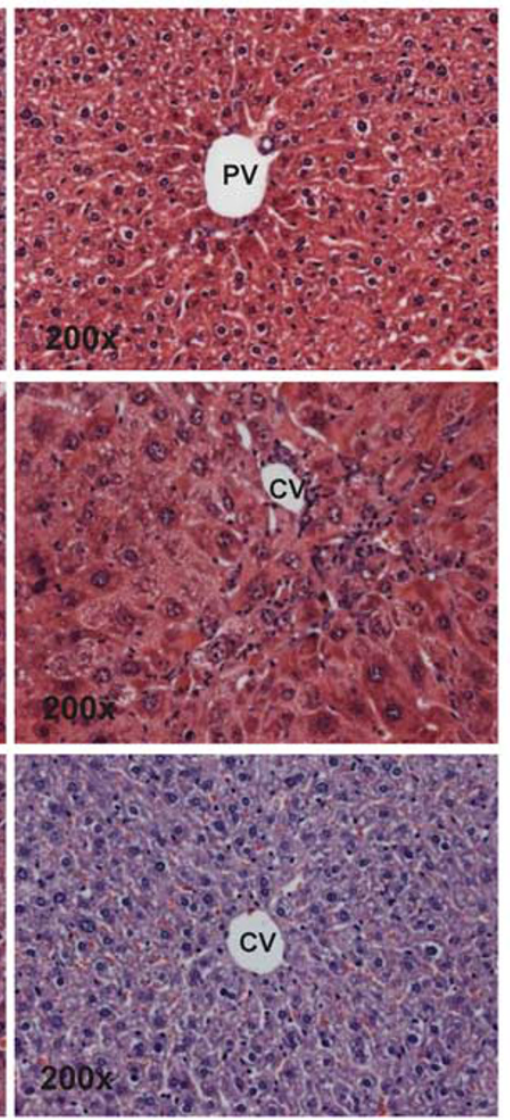

b
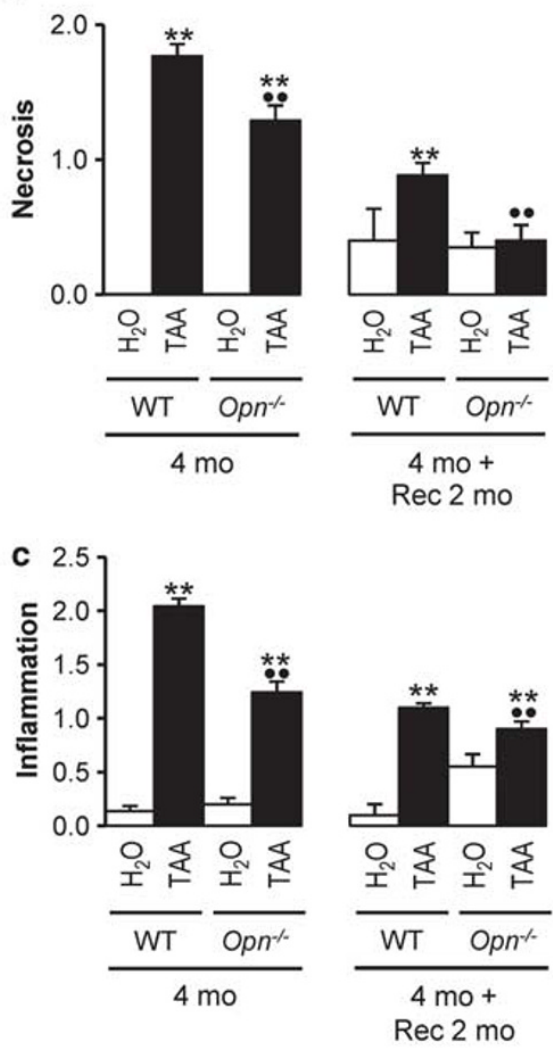

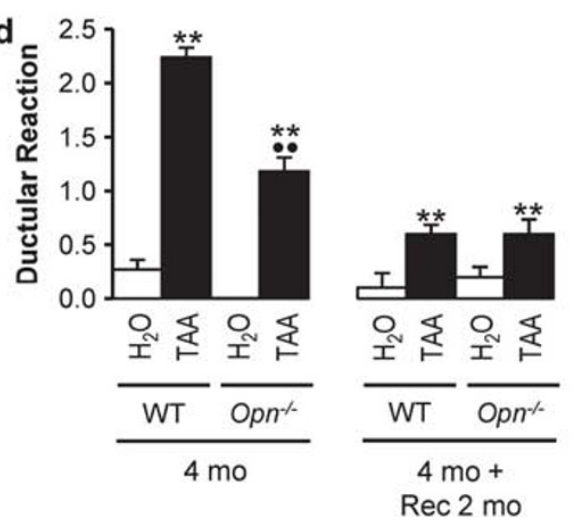

Figure 1 Opn ablation accelerates recovery from TAA-induced liver injury in mice. C57BL/6 J WT and Opn ${ }^{-/-}$mice were administered TAA in the drinking water or iso-volumetric amounts of water for 4 months. TAA was then withdrawn for 2 months, and the extent of liver injury was determined. H\&E staining shows more centrilobular and parenchymal necrosis, centrilobular and parenchymal inflammation, and ductular reaction in WT than in $\mathrm{Opn}^{-/-}$mice after 4 months of TAA treatment. In the resolution phase, all parameters of liver injury are lower in both groups of mice; yet, Opn ${ }^{-/-}$ mice show no necrosis compared with WT mice (a). The pathology scores for necrosis, inflammation, and ductular reaction are shown (b-d). The percentage of clearance of necrosis, inflammation, and ductular reaction is shown (e). Results are expressed as average \pm s.e.m., $n=8$; ${ }^{* *} P<0.01$ for TAA vs water; ${ }^{\bullet} P<0.01$ for $O p n^{-/-}+$TAA vs WT + TAA.

scanner (Fujifilm, Stamford, CT). The intensity of the Western blot bands was quantified using the NIH ImageJ software. All samples from the same experiment were run on the same gel and transferred onto the same nitrocellulose membrane. All extracellular proteins analyzed by Western blot were corrected by total protein content, and protein loading was subsequently verified by Ponceau red staining on each nitrocellulose membrane. In every experiment, the loading control used was Calnexin.

\section{Statistical Analysis}

Data were analyzed by a two-factor ANOVA, and results are expressed as mean \pm s.e.m., $n=8$ mice per group, according to the treatment and time point used. 
a
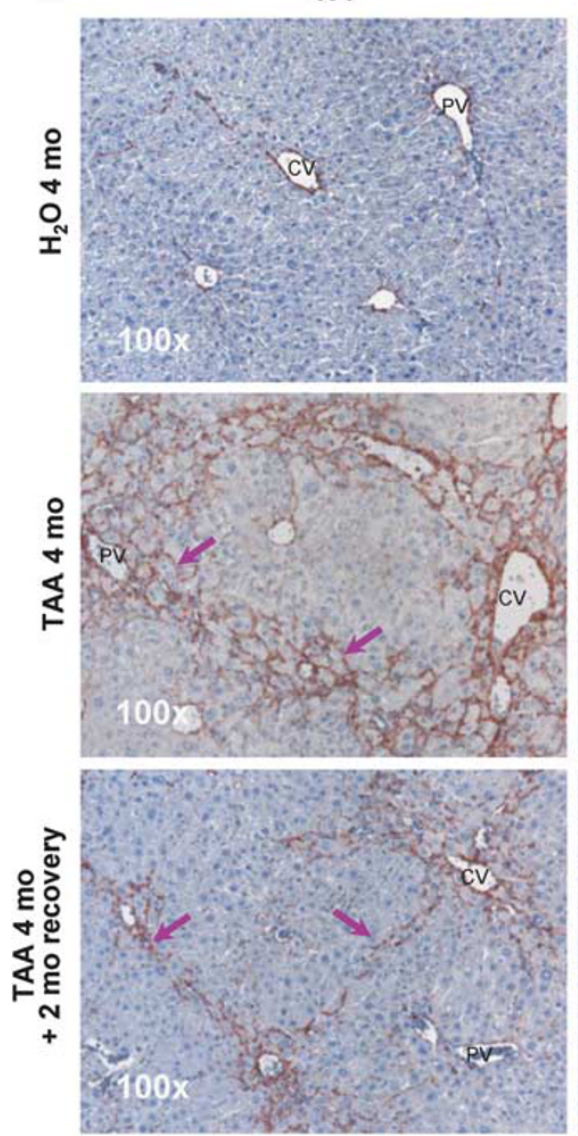
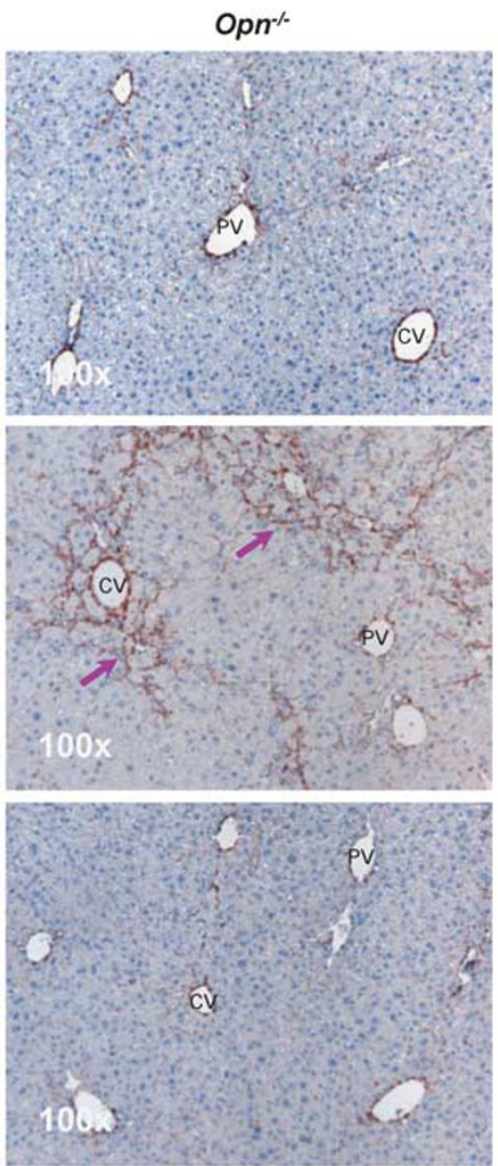

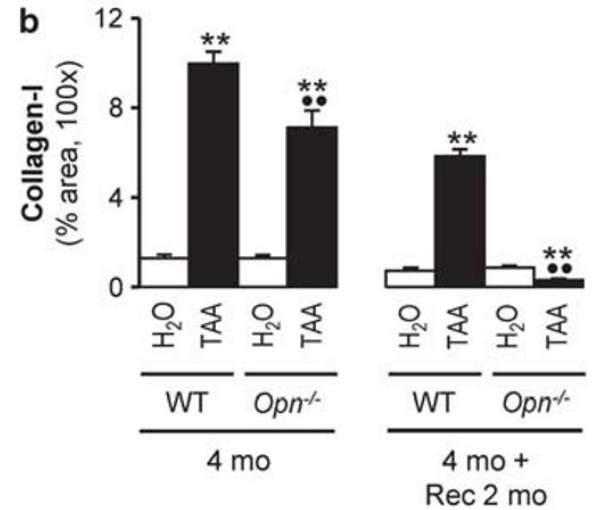

C

\begin{tabular}{cr}
\hline \multicolumn{2}{l}{ Rate of resolution in $2 \mathrm{mo}$} \\
\hline WT & $41 \pm 5.2 \%$ \\
Opn ${ }^{-}$ & $96 \pm 1.1 \%{ }^{*}$ \\
\hline
\end{tabular}

Figure 2 Opn ablation enhances fibrillar collagen-I removal following TAA-induced liver injury in mice. C57BL/6 J WT and Opn ${ }^{-/-}$mice were administered TAA in the drinking water or iso-volumetric amounts of water for 4 months. TAA was then withdrawn for 2 months, and collagen-I expression was evaluated. Chronic TAA administration increases liver fibrillar collagen-I staining more in WT mice than in Opn ${ }^{-\prime-}$ mice. Withdrawal of TAA for 2 months reduces fibrillar collagen-I content more in Opn ${ }^{-1-}$ mice than in WT mice (pink arrows) (a). Morphometric assessment of the fibrillar collagen-I positively stained areas (b). The rate of resolution is expressed as the percentage of reduction in fibrillar collagen-I staining in the resolution phase compared with that at 4 month of TAA treatment for each genotype (c). Results are expressed as average \pm s.e.m., $n=8$; $* * P<0.01$ for TAA $v s$ water; $\bullet P<0.01$ for $O p n^{-/-}+$TAA vs WT + TAA.

\section{RESULTS}

\section{Opn Ablation Accelerates Recovery and ECM} Remodeling Following TAA-Induced Liver Injury in Mice WT and $O p n^{-1-}$ mice were treated with TAA for 4 months following which half of the mice were killed to assess the extent of liver injury at the peak of the treatment, and the other half were allowed to recover from liver fibrosis for 2 months. Overall, $\mathrm{Opn}^{-1-}$ mice had no centrilobular and parenchymal necrosis after a 2-month recovery, whereas WT mice showed a necrosis score of 1 as shown by H\&E staining (Figures $1 \mathrm{a}$ and $\mathrm{b}$ ). Yet, there was similar centrilobular and parenchymal inflammation (Figures $1 \mathrm{a}$ and $\mathrm{c}$ ) along with moderate ductular reaction in both groups of mice (Figures la and d). There was significant improvement in necrosis in the recovered $\mathrm{Opn}^{-1-}$ mice compared with WT mice (Figure 1e). TAA administration for 4 months increased plasma ALT activity more in WT (control vs TAA: $39 \pm 4$ vs $192 \pm 10 \mathrm{IU} / \mathrm{l})$ than in $\mathrm{Opn}^{-/-}$mice $(35 \pm 3$ vs $61 \pm 9 \mathrm{IU} / \mathrm{l})$; yet, although WT still showed slightly elevated ALT following recovery, it was normal in $\mathrm{Opn} n^{-/-}$mice.

\section{Opn Deletion Enhances Collagen-I Removal Following TAA-Induced Liver Injury in Mice}

As fibrillar collagen-I is the key component of the ECM in the fibrotic liver, thus, we determined the amount of fibrillar collagen-I in these livers by specific IHC. Chronic TAA treatment increased the amount of fibrillar collagen-I in WT mice and in $\mathrm{Opn}^{-1-}$ mice. The total content of fibrillar collagen-I in $\mathrm{Opn}^{-1-}$ mice was much lower than that in WT mice (Figures 2a and b). After 2 months of recovery, 96\% of the fibrillar collagen-I was cleared in $\mathrm{Opn}^{-/-}$mice compared with only $41 \%$ of clearance in WT mice (Figure 2c). Overall, $\mathrm{Opn}^{-1-}$ showed decreased content of collagenous proteins $(2.1 \pm 0.2)$, whereas WT mice still had high amount of collagenous proteins $(3.7 \pm 0.4)$ as quantified by morphometric analysis of Sirius red/fast green staining (Figures 
a
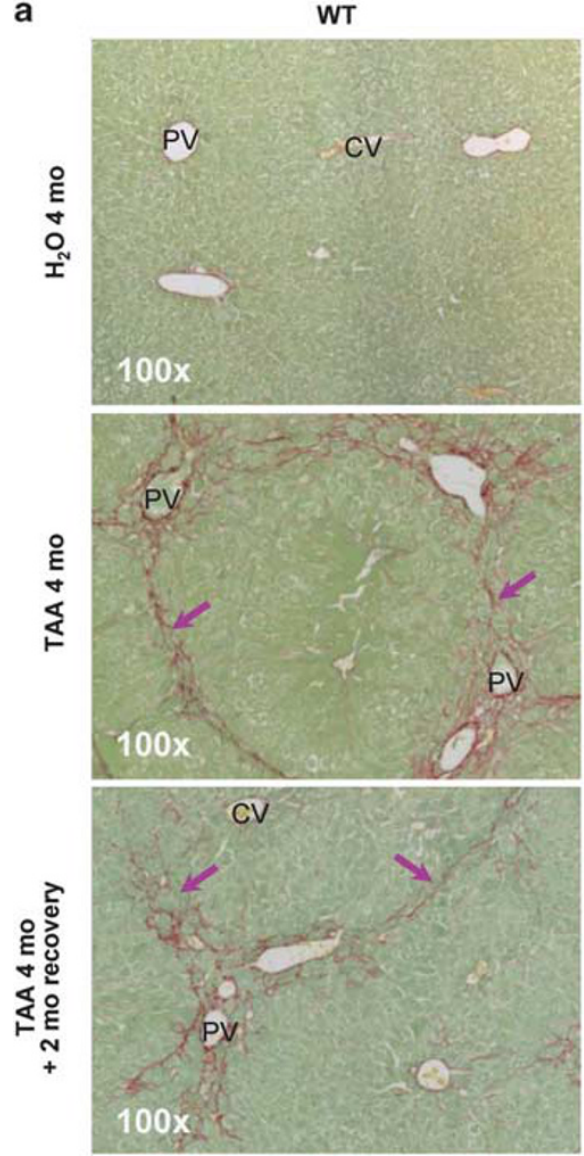
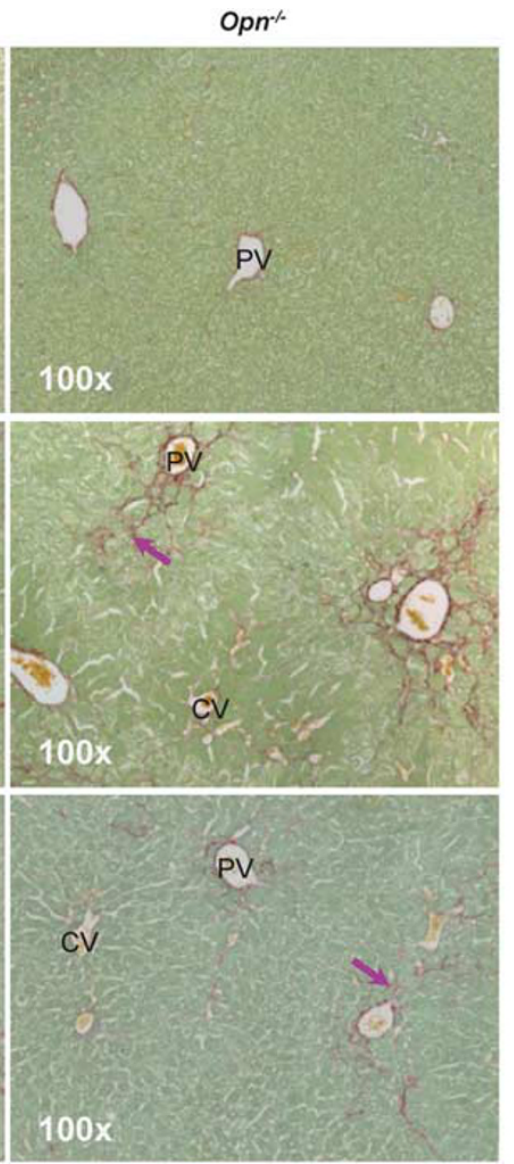

b

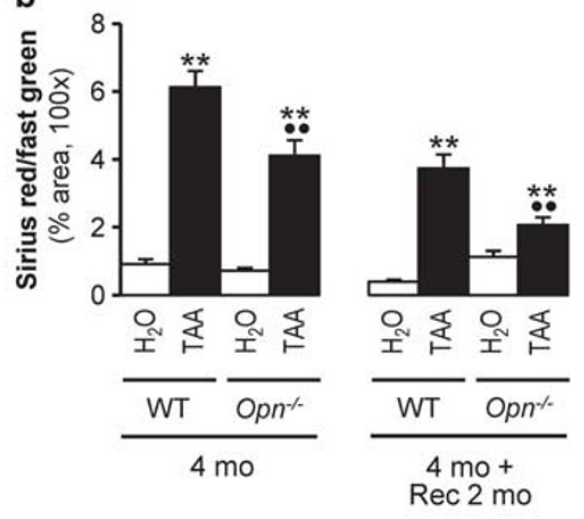

Figure 3 Opn ablation increases ECM remodeling following TAA-induced liver injury in mice. C57BL/6 J WT and Opn ${ }^{-/-}$mice were administered TAA in the drinking water or iso-volumetric amount of water for 4 months. TAA was then withdrawn for 2 months, and the amount of total collagenous proteins was determined. Sirius red/fast green staining shows more total collagenous proteins in WT compared with Opn ${ }^{-/-}$TAA-treated mice (pink arrows) (a). Morphometric assessment of the Sirius red/fast green-stained areas (b). Results are expressed as average \pm s.e.m., $n=8$; $* *<0.01$ for TAA vs water; ${ }^{\bullet P}<0.01$ for Opn ${ }^{-1-}+$ TAA vs WT + TAA.

$3 a$ and $b$ ). Hence, deletion of OPN promotes pathological fibrillar collagen-I clearance.

\section{Opn $^{-/-}$Mice Show Lower TIMP-1 Expression Than WT Mice Following TAA-Induced Liver Injury in Mice}

As MMPs and TIMPs are key enzymes regulating ECM remodeling under physiological and non-physiological conditions, they are key for the resolution of liver fibrosis due to their ability to condition the rate of ECM degradation. Thus, we measured their expression in the livers from these mice. Chronic TAA treatment induced the expression of TIMP-1 and MMP-13 in both groups of mice; yet, only TIMP-1 considerably decreased following resolution of liver fibrosis in $\mathrm{Opn}^{-/-}$mice, which could explain why even in the absence of an overt change in MMP-13 protein expression there may be less inhibition of MMP-13 activity in $\mathrm{Opn}^{-1-}$ mice recovering from TAA treatment. Both MMP-2 and MMP-9 were similarly downregulated in both groups of recovering mice (Figure $4 \mathrm{a}$ ). More $\alpha$ SMA-positive cells were found in TAA-treated WT mice than in $\mathrm{Opn}^{-1-}$ mice. After
TAA withdrawal for 2 months, no $\alpha$ SMA-positive cells were detected in WT and $\mathrm{Opn}^{-/-}$mice (Figure $4 \mathrm{~b}$ ).

\section{Opn ${ }^{-1-}$ Mice Show Similar Number of Activated HSCs Than WT Mice Following TAA-Induced Liver Injury in Mice}

As activated HSCs are the major source of matrix and ECM-modulating enzymes, next, we analyzed their activation state in these mice by performing immunohistochemical analysis for $\alpha$-SMA, a specific marker for HSC activation. After 2 months of TAA withdrawal, the expression of $\alpha$-SMA in WT and in $\mathrm{Opn}^{-1-}$ mice was similar, suggesting that differences in collagen-I expression between these two groups of mice may not be related to HSC clearance (Figure 4).

\section{DISCUSSION}

Liver repair is a complex process, and it has been known for some time that healing can be either regenerative or scar forming. It is now clear that, although permanent scar takes 
a

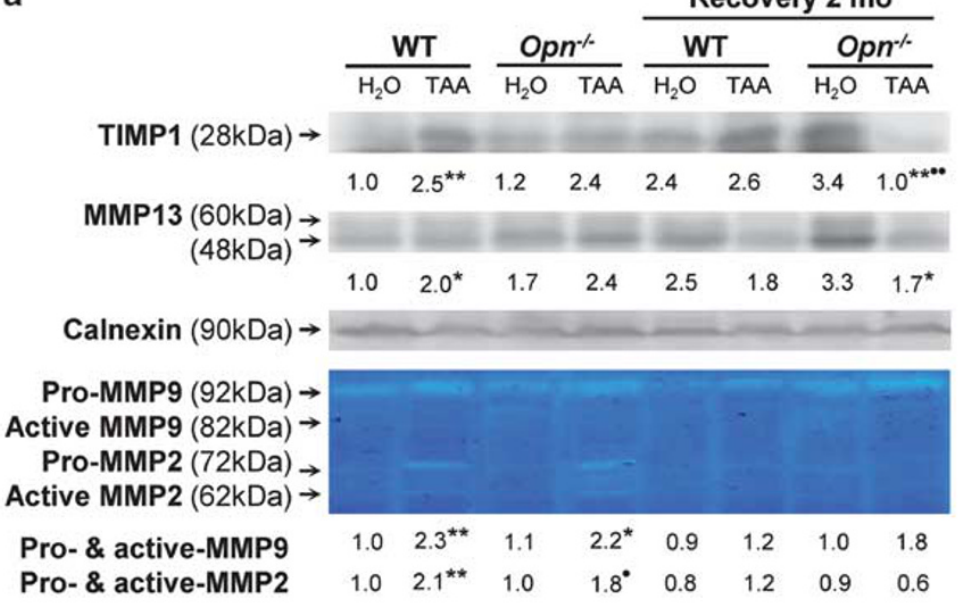

b

WT

$\mathrm{Opn}^{\%}$
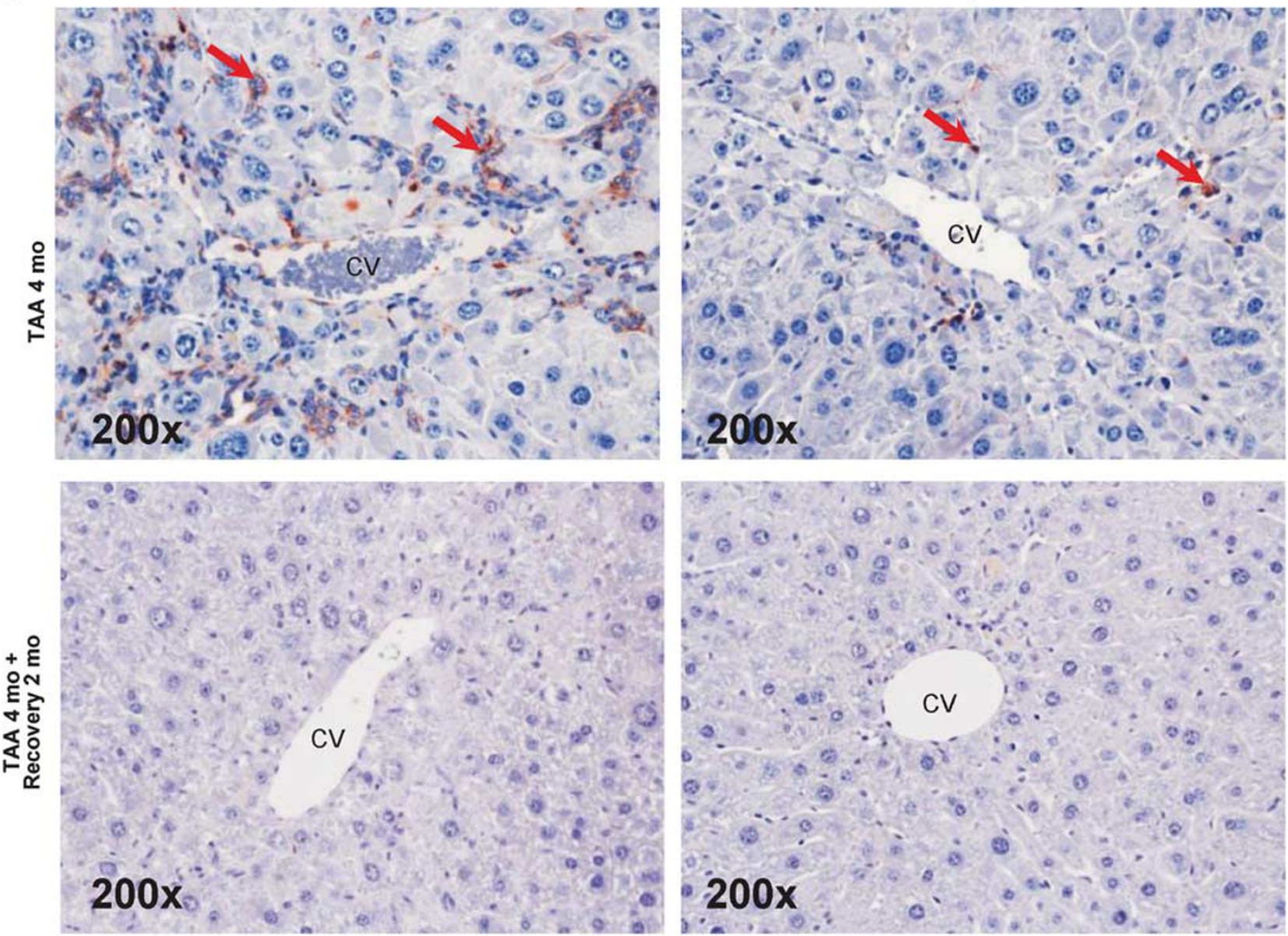

Figure $4 \mathrm{Opn}^{-/-}$mice show lower TIMP-1 expression than WT mice following TAA-induced liver injury in mice. C57BL/6 J WT and Opn ${ }^{-/-}$mice were administered TAA in the drinking water or iso-volumetric amounts of water for 4 months. TAA was then withdrawn for 2 months, and protein expression was analyzed by western blot. MMP-13, MMP-2, MMP-9, and TIMP-1 expressions increase after chronic TAA treatment in WT and Opn ${ }^{-}-$ mice. After 2 months of recovery, Opn ${ }^{-1-}$ mice show significantly lower TIMP-1 expression than WT mice. A representative western blot is shown (a). IHC for $\alpha$-SMA (red arrows) (b). Results are expressed as average \pm s.e.m., $n=8$; ${ }^{*} P<0.05$ and ${ }^{* *} P<0.01$ for TAA vs water; ${ }^{\bullet} P<0.05$ and ${ }^{\bullet \bullet} P<0.01$ for $\mathrm{Opn}^{-1-}+$ TAA vs WT + TAA.

long time to mature, yet the process is rather coordinated and very dynamic in nature. There is an increasing acceptance that liver fibrosis that occurs as a consequence of these events is also dynamic and therefore responsive to reversal. Thus, targeting key participants to achieve a state favoring regenerative repair should provide a great opportunity to not 
only beneficially influence outcome in repair but also to affect reversal of fibrosis.

In addition to preventing or slowing down the mechanisms driving fibrogenesis, considerable amount of research in this field is now focusing on identifying the mediators driving fibrosis resolution to treat patients with fibrosis or even cirrhosis. Fibrosis resolution can be achieved by reducing ECM deposition, by active degradation of pathological fibrillar collagen-I protein, and by decreasing the number of pro-fibrogenic cells.

Previous work from our laboratory identified OPN as an emergent soluble matricellular cytokine and ECM-bound molecule promoting liver fibrosis. ${ }^{17}$ Moreover, the cell- and matrix-binding ability of OPN also appears to facilitate proper stromal and fibrillar collagen network organization. We proposed and demonstrated that OPN drives the fibrogenic response, among others, by directly regulating collagen-I deposition. ${ }^{17}$ Therefore, because of the direct effects of OPN on regulating collagen-I protein expression, in this study, we hypothesized that OPN could also delay fibrosis resolution. To demonstrate this, we compared the rate of fibrosis resolution, with particular attention to fibrillar collagen-I, following chronic TAA treatment in WT mice and in $\mathrm{Opn}^{-/-}$mice.

We previously demonstrated that chronic TAA treatment increases OPN expression in the mouse liver. ${ }^{17} \mathrm{WT}$ mice and $\mathrm{Opn}^{-1-}$ mice were chronically administered TAA for 4 months to let liver injury peak and then half of the mice were allowed to recover for 2 months by removing TAA from the drinking water. In the resolution phase, WT mice and $\mathrm{Opn}^{-/-}$mice showed similar centrilobular and parenchymal inflammation and ductular reaction; yet, $\mathrm{Opn}^{-1-}$ mice showed no necrosis compared with WT mice, which may contribute to faster fibrosis resolution. Moreover, the extent of fibrosis was reduced in both groups of mice. However, $\mathrm{Opn}^{-1-}$ mice presented complete fibrosis regression compared with WT mice. ECM remodeling was particularly remarkable for fibrillar collagen-I, which was almost absent in $\mathrm{Opn}^{-1-}$ mice, whereas WT mice still showed fibrosis stage-2. Thus, Opn ablation allows significant pathological fibrillar collagen-I removal during the resolution of liver fibrosis.

Fibrosis results from tipping the balance between the ECM degradation and synthesis by inhibiting the ECM degradation enzymes and increasing their inhibitors; this, in turn, favors ECM accumulation (ie, collagen-I) in the injured liver. Next, to dissect how this occurred, we examined the expression of the major ECM-modulating enzymes, MMPs and their inhibitors, TIMPs, both highly expressed in activated HSCs. Although there was no significant difference in TIMP-1 expression and MMP-2 activity between WT and $O p n^{-1-}$ mice after 4 months of TAA treatment, yet, following the resolution phase, $\mathrm{Opn}^{-/-}$mice had lower TIMP-1 expression compared with WT mice, which could allow greater MMP-13 activity even if MMP-13 protein expression was not further induced. The decrease in TIMP-1 in $\mathrm{Opn}^{-/-}$mice after fibrosis resolution may reflect a faster resolution rate, restoration to normal liver function driving ECM-modulating enzymes back to their baseline activity. Hence, less TIMP1 expression may facilitate HSCs clearance, ${ }^{13,15}$ reduce ECM deposition, and promote fibrosis resolution in $\mathrm{Opn}^{-/-}$mice compared with WT mice.

As activated HSCs are a major source of ECM, mostly collagen-I in liver injury, ${ }^{8}$ next, we performed $\alpha$-SMA immunohistochemical analysis to evaluate HSCs activation. More $\alpha$-SMA-positive cells were observed in WT than in $\mathrm{Opn}^{-/-}$mice at 4 months of TAA treatment; however, the number of $\alpha$-SMA-positive cells decreased equally both in WT and in $\mathrm{Opn}^{-1-}$ mice during the resolution phase. The clearance of HSC did not account for the difference in collagen-I expression between the two groups of mice.

In conclusion, although the pro-fibrogenic effects of OPN have been demonstrated in several tissues including the liver, ${ }^{17,26-29}$ the role of OPN in the resolution of liver fibrosis was not explored until now. This study suggests that deletion of the Opn gene induces faster removal of fibrillar collagen-I after chronic liver injury likely due to a direct action on TIMP-1 expression and to less activated HSCs.

\section{ACKNOWLEDGEMENTS}

This study was supported by Postdoctoral Fellowship from Keio University School of Medicine (NK); US Public Health Service Grants 5 R01 DK069286, 2 R56 DK069286, and 3 R56 DK069286-06S1 from the National Institute of Diabetes and Digestive and Kidney Diseases (NN); US Public Health Service Grant U01 AA021887-01 from the National Institute on Alcohol Abuse and Alcoholism (NN); and US Public Health Service Grants 5 P20 AA017067, 5 P20 AA017067-01S1, and 5 P20 AA017067-03S1 from the National Institute on Alcohol Abuse and Alcoholism (NN and MIF).

\section{DISCLOSURE/CONFLICT OF INTEREST}

The authors declare no conflict of interest.

1. Mormone E, George J, Nieto N. Molecular pathogenesis of hepatic fibrosis and current therapeutic approaches. Chem Biol Interact 2011;193:225-231.

2. Daley WP, Peters SB, Larsen M. Extracellular matrix dynamics in development and regenerative medicine. J Cell Sci 2008;121(Pt 3): 255-264.

3. Iredale JP. Hepatic stellate cell behavior during resolution of liver injury. Semin Liver Dis 2001;21:427-436.

4. Wanless IR, Nakashima E, Sherman M. Regression of human cirrhosis. Morphologic features and the genesis of incomplete septal cirrhosis. ArchPathol Lab Med 2000;124:1599-1607.

5. Chang TT, Liaw YF, Wu SS, et al. Long-term entecavir therapy results in the reversal of fibrosis/cirrhosis and continued histological improvement in patients with chronic hepatitis B. Hepatology 2010; 52:886-893.

6. Hammel P, Couvelard A, O'Toole D, et al. Regression of liver fibrosis after biliary drainage in patients with chronic pancreatitis and stenosis of the common bile duct. N Engl J Med 2001;344:418-423.

7. Kisseleva T, Brenner DA. Hepatic stellate cells and the reversal of fibrosis. J Gastroenterol Hepatol 2006;21(Suppl 3):S84-S87.

8. Friedman SL. Mechanisms of disease: Mechanisms of hepatic fibrosis and therapeutic implications. Nat Clin Pract Gastroenterol Hepatol 2004;1:98-105.

9. Arthur MJ, Fibrogenesis II. Metalloproteinases and their inhibitors in liver fibrosis. Am J Physiol Gastrointest Liver Physiol 2000;279: G245-G249. 
10. Bedossa P, Paradis V. Liver extracellular matrix in health and disease. J Pathol 2003;200:504-515.

11. Issa R, Williams $\mathrm{E}$, Trim $\mathrm{N}$, et al. Apoptosis of hepatic stellate cells: involvement in resolution of biliary fibrosis and regulation by soluble growth factors. Gut 2001;48:548-557.

12. Chor SY, Hui AY, To KF, et al. Anti-proliferative and pro-apoptotic effects of herbal medicine on hepatic stellate cell. J Ethnopharmacol 2005;100:180-186.

13. Elsharkawy AM, Oakley F, Mann DA. The role and regulation of hepatic stellate cell apoptosis in reversal of liver fibrosis. Apoptosis 2005;10: 927-939.

14. Watson MR, Wallace K, Gieling RG, et al. NF-kappaB is a critical regulator of the survival of rodent and human hepatic myofibroblasts. J Hepatol 2008;48:589-597.

15. Murphy FR, Issa R, Zhou X, et al. Inhibition of apoptosis of activated hepatic stellate cells by tissue inhibitor of metalloproteinase- 1 is mediated via effects on matrix metalloproteinase inhibition: implications for reversibility of liver fibrosis. J Biol Chem 2002;277: 11069-11076.

16. Yoshiji H, Kuriyama S, Yoshii J, et al. Tissue inhibitor of metalloproteinases-1 attenuates spontaneous liver fibrosis resolution in the transgenic mouse. Hepatology 2002;36(4 Pt 1):850-860.

17. Urtasun R, Lopategi A, George J, et al. Osteopontin, an oxidant stress sensitive cytokine, up-regulates collagen-I via integrin alpha(V)beta(3) engagement and PI3K/pAkt/NFkappaB signaling. Hepatology 2012;55:594-608.

18. Cubero FJ, Nieto N. Ethanol and arachidonic acid synergize to activate Kupffer cells and modulate the fibrogenic response via tumor necrosis factor alpha, reduced glutathione, and transforming growth factor beta-dependent mechanisms. Hepatology 2008;48:2027-2039.

19. Nieto N. Oxidative-stress and IL- 6 mediate the fibrogenic effects of [corrected] Kupffer cells on stellate cells. Hepatology 2006;44:1487-1501.
20. Nieto N. Ethanol and fish oil induce NFkappaB transactivation of the collagen alpha2(I) promoter through lipid peroxidation-driven activation of the PKC-PI3K-Akt pathway. Hepatology 2007;45:14331445.

21. Nieto N, Cederbaum Al. Increased Sp1-dependent transactivation of the LAMgamma 1 promoter in hepatic stellate cells co-cultured with HepG2 cells overexpressing cytochrome P450 2E1. J Biol Chem 2003;278:15360-15372.

22. Nieto N, Friedman SL, Cederbaum Al. Stimulation and proliferation of primary rat hepatic stellate cells by cytochrome P450 2E1-derived reactive oxygen species. Hepatology 2002;35:62-73.

23. Urtasun $\mathrm{R}$, Cubero FJ, Vera $\mathrm{M}$, et al. Reactive nitrogen species switch on early extracellular matrix remodeling via induction of MMP1 and TNFalpha. Gastroenterology 2009;136:1410-1422, e1411-1414.

24. Liaw $L$, Birk DE, Ballas $C B$, et al. Altered wound healing in mice lacking a functional osteopontin gene (spp1). J Clin Invest 1998;101: 1468-1478.

25. Kleiner DE, Brunt EM, Van Natta $M$, et al. Design and validation of a histological scoring system for nonalcoholic fatty liver disease. Hepatology 2005;41:1313-1321.

26. Takahashi F, Takahashi K, Okazaki T, et al. Role of osteopontin in the pathogenesis of bleomycin-induced pulmonary fibrosis. Am J Respir Cell Mol Biol 2001;24:264-271.

27. Wu M, Schneider DJ, Mayes MD, et al. Osteopontin in systemic sclerosis and its role in dermal fibrosis. J Invest Dermatol 2012;132: 1605-1614.

28. Sahai A, Malladi P, Melin-Aldana $H$, et al. Upregulation of osteopontin expression is involved in the development of nonalcoholic steatohepatitis in a dietary murine model. Am J Physiol Gastrointest Liver Physiol 2004;287:G264-G273.

29. Lee SH, Seo GS, Park YN, et al. Effects and regulation of osteopontin in rat hepatic stellate cells. Biochem Pharmacol 2004;68:2367-2378. 